\title{
The value of lab findings in early diagnosis of acute mesenteric ischemia
}

\author{
Rouzbeh Rajaei Ghafouri', Saeed Shahbazi ${ }^{1}$, Changiz Gholipour ${ }^{2}$, Samad Shams-Vahdati ${ }^{3}$, Manouchehr \\ Khoshbaten ${ }^{4}$, Amir Ghaffarzad', Respina Jalilian ${ }^{5 *}$ \\ 'Department of Emergency Medicine, Tabriz University of Medical Sciences, Tabriz, Iran \\ ${ }^{2}$ Road Traffic Injury Research Center, Department of Surgery, Tabriz University of Medical Sciences, Tabriz, Iran \\ ${ }^{3}$ Road Traffic Injury Research Center, Department of Emergency Medicine, Tabriz University of Medical Sciences, Tabriz, Iran \\ ${ }^{4}$ Liver and Gastrointestinal Disease Research Center, Tabriz University of Medical Sciences, Tabriz, Iran \\ ${ }^{5}$ Road Traffic Injury Research Center, Tabriz University of Medical Sciences, Tabriz, Iran
}

\author{
Received: 20 October 2015 \\ Accepted: 30 November 2015 \\ Published online: 5 December 2015 \\ * Corresponding author: Respina \\ Jalilian, Imam Reza teaching hospital, \\ Golgasht Ave, Tabriz, Iran. \\ Email: respina.jalilian@gmail.com \\ Competing interests: The authors \\ declare that there are no conflict of \\ interest. \\ Funding information: None. \\ Citation: Rajaei Ghafouri R, Shahbazi \\ S, Gholipour C, Shams-Vahdati S, \\ Khoshbaten M, Ghaffarzad A, et al. The \\ value of lab findings in early diagnosis \\ of acute mesenteric ischemia. Journal \\ of Emergency Practice and Trauma \\ 2016; 2(2): 46-49. doi: 10.15171/ \\ jept.2015.20.
}

\begin{abstract}
Objective: Acute mesenteric ischemia (IMA) is a vascular emergency with broad variability of clinical presentations and non-specific laboratory findings. Therefore, there is a significant need for reliable serological markers of ischemia. Various laboratory studies may be performed for suspected AMI, but in general, such studies will not establish the diagnosis.

Methods: In a prospective, non-interventional study, from October 2012 to October 2013, we investigated 70 patients with probable diagnosis of AMI. Blood samples were taken from patients and analyzed for complete blood count (CBC), prothrombin time (PT), partial thromboplastin time (PTT), international normalized ratio (INR), urea, creatinine $(\mathrm{Cr})$, sodium $(\mathrm{Na})$, potassium $(\mathrm{K})$, D-dimer, lactate, amylase, $\mathrm{PH}$, partial pressure of carbon dioxide $\left(\mathrm{PCO}_{2}\right)$, and bicarbonate $\left(\mathrm{HCO}_{3}\right)$. Finally the results were compared with $\mathrm{AMI}$ diagnosis confirmed by computed tomography (CT) angiography.

Results: Seventy patients with acute severe abdominal pain were studied. Thirty-nine patients $(55.7 \%)$ were male and 31 patients $(44.3 \%)$ were female. The average age was $68.01 \pm 14.67( \pm S D)$. Based on CT-angiography results, 27 (38.6\%) patients had AMI and $43(61.4 \%)$ patients did not have AMI. Chi-squire test showed $P$ values of 0.606 and 0.986 for relations between sex and risk factors with AMI correspondingly. One-sample Kolmogorov-Smirnov revealed white blood cell (WBC), hemoglobin $(\mathrm{Hb})$, platelets (PIt), urea, $\mathrm{Cr}, \mathrm{Na}, \mathrm{K}, \mathrm{PCO}_{2}$ and $\mathrm{HCO}_{3}$ as normally distributed parameters $(P>0.05)$. Moreover $\mathrm{PT}$, PTT, INR, D-dimer, lactate, amylase, and PH were non-normally distributed $(P<0.05)$.

Conclusion: We found a significant relation between increased serum lactate level and definitive AMI diagnosis. We recommend rising serum lactate as a finding in AMI.

Keywords: Mesenteric ischemia, Fibrin fragment D, Lactate, Early diagnosis
\end{abstract}

\section{Introduction}

Acute mesenteric ischemia (AMI) is a life threatening disease and one of the emergency surgery conditions (1). A gradual obstruction, known as chronic mesenteric ischemia does not cause a serious problem because of formation of collateral vessels. However, an acute obstruction of either main mesenteric arteries or even one of the branches causes severe symptoms and needs early intervention (2). Four causes of abdominal ischemia exist including: 1) acute mesenteric arterial embolism, 2) acute mesenteric arterial thrombosis, 3) non-occlusive mesenteric ischemia (systemic hypoperfusion from cardiac failure, sepsis, medications), and 4) mesenteric venous thrombosis (3). Regardless of pathophysiologic cause, AMI leads to the intestinal mucosa flaking after 3 hours of start and whole intestinal thickness infarction after 6 hours and finally perforation and peritonitis (2). Clinically, the most common symptom remains a severe abdominal pain that is not proportional to the amount of tenderness. This pain is typically in colic form and localized in umbilical region. There may be other associated symptoms like nausea, vomiting, and diarrhea. Physical exam in early stage only reveals a mild tenderness (3). Early diagnosis of this condition is difficult. Procedures like plain abdominal x-ray, abdominal ultrasonography, and computed tomography (CT) scan are performed to rule out the other differential diagnosis and are often normal especially in early stages. In patients with unstable hemodynamic and peritonitis symptoms, the first procedure is emergency laparotomy $(1,2)$. Angiography of mesenteric arteries is the most reli- 
able diagnostic method with the sensitivity of $74 \%-100 \%$ and specificity of $100 \%$. Because of difficult access, high costs and wasting time in this method, researchers try to find a laboratory method for early diagnosis of AMI (4). Delayed lab findings include: leukocytosis, acidosis, increased amylase, and increased creatinine kinase (BB isoenzyme). Normal serum level of leukocyte and D-dimer in primary phase rules out AMI diagnosis (5). Due to the broad variability of clinical presentations and nonspecific laboratory findings the mortality of this condition remains high, thus there is a significant need for reliable and enhanced serological markers of intestinal ischemia. Several studies have shown the role of different serum biomarkers in early diagnosis of AMI but none of them is sufficiently accurate and singly reliable (6).

\section{Methods}

In a prospective, non-interventional study that was conducted in the emergency department of Imam Reza hospital, Tabriz, Iran, from October 2012 to October 2013, we investigated 70 patients with probable diagnosis of AMI. All patients with a severe abdominal pain, which was disproportionate with tenderness and one of the AMI risk factors such as: atrial fibrillation, hear failure, ischemic heart disease, hypertension and diabetes were included (7). Blood samples were taken from patients and analyzed for complete blood count (CBC), prothrombin time (PT), partial thromboplastin time (PTT), international normalized ratio (INR), urea, creatinine $(\mathrm{Cr})$, sodium $(\mathrm{Na})$, potassium (K), D-dimer, lactate, amylase, $\mathrm{PH}$, partial pressure of carbon dioxide $\left(\mathrm{PCO}_{2}\right)$, and bicarbonate $\left(\mathrm{HCO}_{3}\right)$. Patients with high possibility conditions of rising $\mathrm{D}$-dimer were excluded (recent history of surgery, trauma, pregnancy, deep vein thrombosis [DVT], etc.). No intervention was done in the course of routine diagnosis and treatment of patients in emergency department. Finally an ex-

Table 1. Frequencies of patients' risk factors

\begin{tabular}{llccccc}
\hline & & \multicolumn{5}{c}{ Risk factors } \\
\cline { 3 - 7 } & & DM & AF & HF & HTN & IHD \\
\hline \multirow{2}{*}{ AMI } & No. & 12 & 9 & 7 & 13 & 9 \\
& $\%$ & 17.1 & 12.85 & 10.0 & 18.5 & 12.9 \\
\multirow{3}{*}{ N ot AMI } & No. & 21 & 16 & 14 & 20 & 13 \\
& $\%$ & 30.0 & 22.85 & 20.0 & 28.6 & 18.5 \\
\multirow{3}{*}{ Total } & No. & 33 & 25 & 21 & 33 & 22 \\
& $\%$ & 47.1 & 35.7 & 30.0 & 47.1 & 31.4 \\
\hline
\end{tabular}

act diagnosis was obtained according to CT-angiography findings and compared with lab results.

We entered all collected data in this study into SPSS (version 15.0; SPSS, Chicago, Ill) software. We used descriptive statics to analyze sex, age, results of CT scan, risk factors and lab findings.

We used chi-squire method to analyze the relationship between risk factors and gender with CT-angiography results. We used one-sample Kolmogorov-Smirnov test to evaluate whether the data were normally distributed or not. Independent $t$ test was used to analyze the normally distributed date. Moreover, Mann-Whitney U test was used to analyze non-normally distributed data. $P$ values $<0.05$ were considered statistically significant.

\section{Results}

In this study, 70 patients were suspected AMI. The results were as follows: Among them, 39 patients (55.7\%) were male and 31 patients $(44.3 \%)$ were female. The average age was $68.01 \pm 14.67( \pm \mathrm{SD})$. Based on CT-angiography results, 27 (38.6\%) patients had AMI and 43 (61.4\%) patients did not have AMI.

Chi-squire test showed $P$ values of 0.606 and 0.986 for relations between sex and risk factors with AMI correspondingly. Frequencies of risk factors are shown in Table 1.

The mean white blood cell (WBC), hemoglobin ( $\mathrm{Hb}$ ), platelets (Plt), PT, PTT, INR, urea, Cr, Na, K, D-dimer, lactate, amylase, $\mathrm{PH}, \mathrm{PCO}_{2}, \mathrm{HCO}_{3}$ were $11.66 \pm 6.61( \pm \mathrm{SD})$, $12.63 \pm 3.08( \pm \mathrm{SD}), 244.66 \pm 119.40( \pm \mathrm{SD}), 15.13 \pm 4.89$ $( \pm$ SD), $36.39 \pm 8.90( \pm$ SD), $1.44 \pm 1.16( \pm$ SD), $55.99 \pm 33.38$ $( \pm \mathrm{SD}), 1.24 \pm 0.36( \pm \mathrm{SD}), 139.04 \pm 4.28( \pm \mathrm{SD}), 0.75 \pm 1.15$ $( \pm S D), \quad 27.55 \pm 13.24 \quad( \pm S D), \quad 102.35 \pm 302.09 \quad( \pm S D)$, $7.31 \pm 0.33( \pm \mathrm{SD}), 36.05 \pm 9.40( \pm \mathrm{SD}), 20.33 \pm 5.26( \pm \mathrm{SD})$ respectively.

One-sample Kolmogorov-Smirnov revealed WBC, $\mathrm{Hb}$, Plt, urea, $\mathrm{Cr}, \mathrm{Na}, \mathrm{K}, \mathrm{PCO}_{2}$ and $\mathrm{HCO}_{3}$ as normally distributed parameters $(P>0.05)$. Moreover, PT, PTT, INR, $\mathrm{D}$-dimer, lactate, amylase and $\mathrm{PH}$ were non-normally distributed $(P<0.05)$. Independent $t$ test results are shown in Table 2.

The mean ranks of PT, PTT, INR, D-dimer, lactate, amylase and $\mathrm{PH}$ in patients with and without AMI were 39.94 and $32.71,41.09$ and $31.99,40.39$ and $32.43,38.96$ and $33.33,43.93$ and $30.21,33.26$ and $36.53,34.65$ and 36.03 respectively. The results of Mann-Whitney $U$ test is shown in Table 3.

Table 2. Results of independent $t$ test

\begin{tabular}{cccccccccc}
\hline & WBC & Hb & PIt & Urea & Cr & Na & K $_{2}$ & HCO $_{3}$ \\
\hline$P$ value & 0.87 & 0.69 & 0.61 & 0.53 & 0.19 & 0.91 & 0.70 & 0.74 & 0.16 \\
\hline
\end{tabular}

Table 3. Results of Mann-Whitney $U$ test

\begin{tabular}{|c|c|c|c|c|c|c|c|}
\hline & \multicolumn{7}{|c|}{ Parameters } \\
\hline & PT & PTT & INR & D-dimer & Lactate & Amylase & PH \\
\hline$P$ value & 0.132 & 0.066 & 0.094 & 0.256 & 0.006 & 0.465 & 0.781 \\
\hline
\end{tabular}




\section{Discussion}

There was no significant relationship between risk factors and gender with AMI. We could not observe any significant relationship between normally distributed data and AMI $(P>0.05)$. Serum lactate levels in patients with AMI (mean rank: 43.93) were significantly higher than patients without AMI (mean rank: 30.21) $(P=0.006)$.

Serum lactate has repeatedly been assumed to be the best marker of AMI. However, numerous studies have proved that it is only an unspecific marker of tissue hypo perfusion and does not confirm AMI diagnosis. A review study by Demir et al has completely evaluated the role for serum lactate measurement in diagnosing AMI. They discovered that despite the emphasis of many studies on lactate increased in AMI, the level of no single serum marker, including serum lactate, is elevated early and specifically enough in the serum to diagnose AMI (8). In some similar studies with close results to current data, serum lactate as a marker of cell hypoxia has been shown to have a high sensitivity of $(96 \%$ or $78 \%)$ in patients with mesenteric ischemia $(9,10)$. On the other hand, low lactate levels may help in ruling out the diagnosis of intestinal ischemia and avoid unnecessary laparotomies (11-13). A study on a pig that his superior mesenteric artery was surgically ligated revealed that peripheral blood tests do not enable the detection of mesenteric ischemia within 4 hours after onset and during this time lactate changes only appear in superior mesenteric vein (14). Aydin et al in a research on rats with superior mesenteric artery occlusion measured serum lactate and D-dimer. They found that blood L-lactate levels began to increase significantly following forth hour of mesenteric ischemia so it seems to be a suitable marker to use in emergency departments for early AMI diagnosis. Conversely about D-dimer, it was revealed that mesenteric ischemia does not cause significantly D-dimer increase and it is not a useful marker for early diagnosis of AMI (15).

We reached the same consequence about $\mathrm{D}$-dimer. In a recent study, D-dimer did not have a significant difference between AMI and non-AMI patients so as for recent data it is not an appropriate marker to decide about AMI. A prospective study on 67 patients with clinical suspicion of AMI showed that detection of serum D-dimer cannot help to differentiate patients with AMI from those with non-AMI and there is no correlation between serum Ddimer levels and the severity of AMI (16). Kulacoglu et al studied on rats, their data did not support the use of blood D-dimer level as an independent parameter in the diagnosis of mesenteric ischemia due to arterial thrombosis (17). Clot fibrinolysis process in blood produces D-dimer from fibrin degradation. Therefore, D-dimer does not normally exist in plasma and it is created when coagulation system becomes activated. Negative result of D-dimer rules out thrombosis. False positive results occur in liver disease, high rheumatoid factor, inflammation, malignancy, trauma, pregnancy and recent surgery $(18,19)$. According to the role of plasma, D-dimer to detect thrombosis, in contrast to our data, numerous studies confirmed D-dimer as an early diagnostic factor for AMI (20-22). Abd-Elazeem and Selim evaluated D-dimer in AMI patients. They concluded that plasma D-dimer $>3.8$ $\mathrm{ug} / \mathrm{ml}$ combined with relevant clinical variables has a high positive predictive value for early identification of patients with AMI and plasma D-dimer $<0.53 \mathrm{ug} / \mathrm{ml}$ has a high negative predictive value for AMI and can be used as an exclusion test. So D-dimer testing should be performed in patients with acute abdomen (23). Another study on patients with a clinical suspicion of AMI estimated the sensitivity (94.7\%) and specificity (78.6\%) of D-dimer testing for the diagnosis of AMI and mentioned it as a marker for early diagnosis in patients for whom we cannot use CT angiography (24).

Amylase and acidosis stand two other markers that are recommended to be measured in diagnostic process of AMI. Elevated levels of amylase and also acidosis in the blood are two non-specific indicators of mesenteric ischemia $(2,3)$. In the current study, we did not find any significant difference between AMI and non-AMI patients in rising serum amylase and acidosis. So, we do not suggest amylase and acidosis as factors to decide about AMI. In the current study, we just found serum lactate rising in patients with AMI and none of the other investigated factors showed a significant correlation.

\section{Limitations}

This study was carried out in one referral center and also with a small study group. Larger study samples are needed to promote the accuracy of our findings.

\section{Conclusion}

No serum marker is sensitive or specific enough to establish or exclude the diagnosis of AMI. We found a significant relation between increased serum lactate level and definitive AMI diagnosis. We recommend rising serum lactate as a finding in AMI.

\section{Acknowledgments}

The authors want to thank all personal of Liver and Gastrointestinal Disease Research Center, Tabriz University of Medical Sciences, Tabriz, Iran.

\section{Ethical issues}

Ethics Committee of the Tabriz University of Medical Sciences approved the study before patients' enrollment..

\section{Authors' contributions}

All authors met authorship criteria based on International Committee of Medical Journal Editors (ICJME).

\section{References}

1. Belkin M, Owens CD, Whittemore AD. Peripheral Arterial Occlusive Disease. In: Townsend CM Jr, Beasuchamp RD, Evers BM, eds. Sabiston Text book of Surgery: The Biological Basis of Modern Surgical Practice. 18th ed. Philadelphia: WB Saunders; 2008. p. 1973-7. 
2. Lin PH, Kougias P, Bechara C. Arterial disease. In: Brunicardi FC, Anderson DK, Billiar TR, eds. Schwartz's Principles of Surgery. 9th ed. New York: McGraw-Hill; 2010. p. 730-6

3. Acosta S, Nilsson T. Current status on plasma biomarkers for acute mesenteric ischemia. J Thromb Thrombolysis 2012; 33(4): 355-61. doi: 10.1007/ s11239-011-0660-z.

4. Chang RW, Chang JB, Longo WE. Update in management of mesenteric ischemia. World J Gastroenterol. 2006; 12(20): 3243-7. doi: 10.3748/ wjg.v12.i20.3243.

5. Gearhart SL. Mesenteric vascular insufficiency. In: Longo DL, Fauci AS, eds. Harrison's Principles of Interal Medicine. 18th ed. New York: McGraw-Hill; 2012. p. 2510-6.

6. Rosero O, Harsányi L, Szijártó A. Acute mesenteric ischemia: do biomarkers contribute to diagnosis. Orv Hetil 2014; 155(41): 1615-23. doi: 10.1556/ oh.2014.30013. [Article in Hungarian].

7. Stone JR, Wilkins LR. Acute mesenteric ischemia. Tech Vasc Interv Radiol 2015; 18(1): 24-30. doi: 10.1053/j.tvir.2014.12.004.

8. Demir IE, Ceyhan GO, Friess H. Beyond lactate: is there a role for serum lactate measurement in diagnosing acute mesenteric ischemia. Dig Surg 2012; 29(3): 226-35. doi: 10.1159/000338086.

9. Lange H, Jackel R. Usefulness of plasma lactate concentration in the diagnosis of acute abdominal disease. Eur J Surg 1994; 160(6-7): 381-4.

10. Gearhart SL, Delaney CP, Senagore AJ, Banbury MK, Remzi FH, Kiran RP, et al. Prospective assessment of the predictive value of alpha-glutathione S-transferase for intestinal ischemia. Am Surg 2003; 69(4): 324-9.

11. Newman TS, Magnuson TH, Ahrendt SA, SmithMeek MA, Bender JS. The changing face of mesenteric infarction. Am Surg 1998; 64(7): 611-6.

12. Klein HM, Lensing $\mathrm{R}$, Klosterhalfen B, Töns C, Günther RW. Diagnostic imaging of mesenteric infarction. Radiology 1995; 197(1): 79-82.

13. Murray MJ, Gonze MD, Nowak LR, Cobb CF. Serum $\mathrm{D}(-)$-lactate levels as an aid to diagnosing acute intestinal ischemia. Am J Surg 1994; 167(6): 575-8.

14. Kurimoto Y, Kawaharada N, Ito T, Morikawa M, Higami T, Asai Y. An experimental evaluation of the lactate concentration following mesenteric ischemia. Surg Today 2008; 38(10): 926-30. doi: 10.1007/ s00595-007-3737-8.

15. Aydin B, Ozban M, Serinken M, Kaptanoglu B, Demirkan NC, Aydin C. The place of D-dimer and L-lactate levels in the early diagnosis of acute mesenteric ischemia. Bratisl Lek Listy 2015; 116(5): 343-50. doi: 10.4149/bll_2015_094.

16. Chiu YH, Huang MK, How CK, Hsu TF, Chen JD, Chern $\mathrm{CH}$, et al. D-dimer in patients with suspected acute mesenteric ischemia. Am J Emerg Med 2009; 27(8): 975-9. doi: 10.1016/j.ajem.2009.06.006.

17. Kulacoglu H, Kocaerkek Z, Moran M, Kulah B, Atay C, Kulacoglu S, et al. Diagnostic value of blood D-dimer level in acute mesenteric ischaemia in the rat: an experimental study. Asian J Surg 2005; 28(2): 131-5. doi: 10.1016/s1015-9584(09)60277-3.

18. Adam SS, Key NS, Greenberg CS. D-dimer antigen: current concepts and future prospects. Blood 2009; 113 (13): 2878-87. doi: 10.1182/blood-2008-06-165845.

19. Brill-Edward P, Lee A. D-dimer testing in the diagnosis of acute venous thromboembolism. Thromb Haemost 1999; 82(2): 688-94.

20. Kurt Y, Akin ML, Demirbas S, Uluutku AH, Gulderen $\mathrm{M}$, Avsar K, et al. D-dimer in the early diagnosis of acute mesenteric ischemia secondary to arterial occlusion in rats. Eur Surg Res 2005; 37(4): 216-9. doi: 10.1159/000087866.

21. Altinyollar H, Boyabatli M, Berberoğlu U. D-dimer as a marker for early diagnosis of acute mesenteric ischemia. Thromb Res 2006; 117(4): 463-7. doi: 10.1016/j.thromres.2005.04.025.

22. Acosta S, Nilsson TK, Björck M. Preliminary study of D-dimer as a possible marker of acute bowel ischaemia. Br J Surg 2001; 88(3): 385-8. doi: 10.1046/j.1365-2168.2001.01711.x.

23. Abd-Elazeem A, Selim T. The role of plasma D-dimer levels in patients with acute mesenteric ischemia. Egypt J Surg 2006; 25: 60-65.

24. Akyildiz H, Akcan A, Oztürk A, Sozuer E, Kucuk C, Karahan I. The correlation of the D-dimer test and biphasic computed tomography with mesenteric computed tomography angiography in the diagnosis of acute mesenteric ischemia. Am J Surg 2009; 197(4): 429-33. doi: 10.1016/j.amjsurg.2008.02.011. 